\title{
Visible Light Catalytic Degradation of Aniline Wastewater over Multishaped BiOBr Microcrystals
}

\author{
Jun Zhang, Huabo Li, Hairui Yao, and Hao Zhang \\ Chemical Engineering \& Pharmaceutics School, Henan University of Science \& Technology, Luoyang 471023, China \\ Correspondence should be addressed to Jun Zhang; j-zhang@126.com
}

Received 5 September 2015; Revised 23 October 2015; Accepted 2 November 2015

Academic Editor: Davide Vione

Copyright (c) 2015 Jun Zhang et al. This is an open access article distributed under the Creative Commons Attribution License, which permits unrestricted use, distribution, and reproduction in any medium, provided the original work is properly cited.

\begin{abstract}
The photooxidation process of aniline-containing simulated wastewater under visible light irradiation over BiOBr microcrystal grains with different shapes was studied. The distinctive surface microstructures of the BiOBr microcrystals, like clustered flower petals and quadrate lamellas, were produced by using imidazole ionic liquid and inorganic bromide as Br sources and by solvothermal and hydrothermal route, respectively. The ionic liquid not only can impact the products' morphology, but also can largely improve the photocatalytic activity of the $\mathrm{BiOBr}$ microcrystals due to the soft templating effect. The top degradation ratio of the aniline wastewater photocatalyzed by lamellar $\mathrm{BiOBr}$ crystals is $23.71 \%$, but the equivalent value photocatalyzed by flower petallike $\mathrm{BiOBr}$ crystals is almost twice as much; that is, it reaches $46.51 \%$. The photodegradation effect and mechanism over differently shaped $\mathrm{BiOBr}$ microcrystals have been compared.
\end{abstract}

\section{Introduction}

With the rapid development of photocatalytic theory and technology, photocatalytic oxidation (PCO), which is actually used for pollution abatement, especially for degradation of pollutants [1-3], has been attracting more and more attention. The PCO technology with such traits as small investment, high efficiency, mild treatment conditions, and no secondary pollution possesses incomparable advantages over other advanced oxidative process (AOPs). Titanium dioxide $\left(\mathrm{TiO}_{2}\right)$, a semiconductor material with photocatalytic activity found in the 1970s, has been verified to have photodegradation ability for azo dyestuffs, and so forth, but it can only absorb ultraviolet light (ca $4-5 \%$ of sunlight) because of its wide band-gap energy $\left(E_{g}=3.2 \mathrm{eV}\right)$. Poor response to visible light and low utilization efficiency of solar energy have limited its application range. $\mathrm{BiOBr}$ is a newly found semiconductor photocatalyst, which has the narrow bandgap energy $\left(E_{g}=2.7 \mathrm{eV}\right)$ and good visible light response due to its unique lamellar structure made up of alternate overlay of $\left[\mathrm{Bi}_{2} \mathrm{O}_{2}\right]$ and $\mathrm{Br}$ atomic layers, and is endowed with better visible light catalytic activity $[4,5]$. Hence, the synthesis and application development concerning $\mathrm{BiOBr}$ has recently aroused broad interest. Different preparation methods and technological conditions can largely influence the microstructure and morphology of the resulting $\mathrm{BiOBr}$ product [6] and then impact its catalytic ability, selectivity, and stability [7]. Ionic liquids possess peculiar composition and structure and not only can be used as reactants, but also can serve as soft templates to regulate and control the product features $[8,9]$. Introduction of some ionic liquids into the preparation process of $\mathrm{BiOBr}$ microcrystals can be expected to give particular $\mathrm{BiOBr}$ product with exceptional shape, property, and photocatalytic ability.

Aniline is a widely used industrial chemical, but it is also one of the recognized contaminants which can badly pollute water and soil. So, intensive study on controlling and removing the aniline-induced pollution is of great importance $[10,11]$. Due to its high chemical stability, aniline is hardly degraded by some conventional approaches. To our knowledge, the photocatalysts used to study aniline degradation are mostly transition metal oxides, such as $\mathrm{TiO}_{2}$ [12] and $\mathrm{ZnO}$ [13]. In addition, the studies involving $\mathrm{TiO}_{2}$ and $\mathrm{ZnO}$ photocatalysis were almost always based on UV light irradiation. Here, we firstly report the research results about visible light catalytic degradation of aniline-containing 
wastewater over multishaped $\mathrm{BiOBr}$ crystal grains, comparing their photodegradation performance.

\section{Experimental Section}

2.1. Chemicals and Instruments. The chemicals bismuth nitrate $\left(\mathrm{Bi}\left(\mathrm{NO}_{3}\right)_{3} \cdot 5 \mathrm{H}_{2} \mathrm{O}\right)$, sodium bromide $(\mathrm{NaBr})$, ethylene glycol $\left(\left(\mathrm{HOCH}_{2}\right)_{2}\right)$, and aniline $\left(\mathrm{C}_{6} \mathrm{H}_{5} \mathrm{NH}_{2}\right)$, purchased from Shanghai Chemical Reagent Company of China, were of analytically pure grade with purity of more than wt. 99\%; the ionic liquid 1-butyl-3-methylimidazolium bromide $\left(\left[\mathrm{C}_{4} \mathrm{mim}\right] \mathrm{Br}\right)$ was customized product with purity of higher than wt. $98 \%$. The phase composition was characterized on a Bruker D8-advanced X-ray diffractometer. The morphology was observed on a Quanta FEG250 scanning electron microscope. The GC-MS instrument (7890A-5975C) was employed to determine the degradation products under the following GC conditions: chromatographic column of DB-5MS UI $(30 \mathrm{~m} \times 0.32 \mathrm{~mm} \times 0.32 \mu \mathrm{m})$, temperature programming (35 to $185^{\circ} \mathrm{C}$ ), and MS conditions: electron impact ionization source $(70 \mathrm{eV})$ and scanning range (45 180 amu). The specific surface area was measured on a NOVA 2200e surface area analyzer. A double-beam ultraviolet-visible spectrophotometer (TU-1901) was used for measuring the optical adsorption properties of the degraded mixtures.

\subsection{Synthesis of Multishaped BiOBr Microcrystals. Under} room temperature and by magnetic stirring, the $\mathrm{Br}$ source solution prepared by dissolving $2 \mathrm{mmol}\left[\mathrm{C}_{4} \mathrm{mim}\right] \mathrm{Br}$ in $20 \mathrm{~mL}$ $\left(\mathrm{HOCH}_{2}\right)_{2}$ was slowly dripped into the fully mixed bismuth nitrate solution consisting of $2 \mathrm{mmol} \mathrm{Bi}\left(\mathrm{NO}_{3}\right)_{3} \cdot 5 \mathrm{H}_{2} \mathrm{O}$ and $20 \mathrm{~mL}\left(\mathrm{HOCH}_{2}\right)_{2}$. The initial colorless mixture gradually changed to light yellow suspension containing some insoluble particles. The resulting suspension was poured in a $100 \mathrm{~mL}$ teflon-lined autoclave with volumetric filling degree of $80 \%$. After tight sealing, the autoclave was put in a drying oven and kept at $423 \mathrm{~K}$ for $12 \mathrm{~h}$. When the solvothermal reaction was over, the autoclave was naturally cooled to ambient temperature. Through centrifugal separation, the isolated solid was in turn washed by water and absolute ethyl alcohol several times, followed by vacuum desiccation at $333 \mathrm{~K}$ for $10 \mathrm{~h}$. A faint yellow powder-like product sample was obtained, which was labeled as BiOBr-IL. Analogously, by using $\mathrm{NaBr}$ as inorganic $\mathrm{Br}$ source to replace ionic liquid $\left[\mathrm{C}_{4} \mathrm{mim}\right] \mathrm{Br}$ and water as a substitute for ethylene glycol, the same procedures as above were also carried out so as to prepare $\mathrm{BiOBr}$ product. Through such an alternative route, the as-prepared faint yellow powder sample was marked as $\mathrm{BiOBr}-\mathrm{SB}$.

\subsection{Evaluation of Visible Light Catalytic Performance of the} $\mathrm{BiOBr}$ Samples. Using BiOBr crystals as catalysts to photocatalytically degrade aniline solution, the related changes of $\mathrm{UV}$-vis absorbance for the reaction mixture can be used for the evaluation of catalytic performance of the $\mathrm{BiOBr}$ samples. The photocatalytic degradation reaction was carried out in a photochemical reaction apparatus (BL-GHX-V, Shanghai). A high pressure xenon lamp with adjustable power was used to provide light source, and the nonvisible parts $(<420 \mathrm{~nm}$

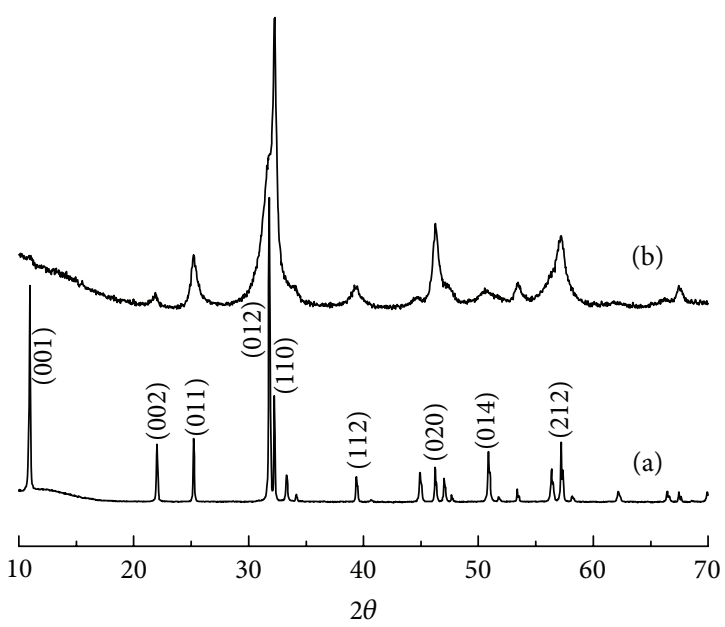

FIGURE 1: The XRD patterns of as-prepared $\mathrm{BiOBr}$ product samples. (a) $\mathrm{BiOBr}-\mathrm{SB}$; (b) BiOBr-IL.

wavelength) were filtered by an optical filter so as to guarantee visible light irradiation. The simulated aniline wastewater with a concentration of $10 \mathrm{mg} / \mathrm{L}$ was obtained by mixing aniline with water. The photocatalytic reaction mixture was prepared by putting $0.05 \mathrm{~g} \mathrm{BiOBr}$ microcrystals in $100 \mathrm{~mL}$ aniline wastewater, which was put in the quartz reaction tube in advance, and magnetically stirring for $60 \mathrm{~min}$ in dark box so as to reach adsorption equilibrium. At this point, the xenon lamp (adjusted at $500 \mathrm{~W}$ ) was turned on, and the lightcatalyzed reaction was immediately activated. Afterwards, $4 \mathrm{~mL}$ reaction solution was sampled every $0.5 \mathrm{~h}$, and the corresponding UV-vis absorption spectrum was measured. According to Lambert-Beer law, the concentration of aniline solution is proportional to absorbance at characteristic absorption wavelength, and the content and degradation ratio of the aniline solution can thereby be quantitatively determined.

\section{Result and Discussion}

3.1. Phase Composition of the BiOBr Samples. The XRD patterns of the $\mathrm{BiOBr}$ samples synthesized by using different $\mathrm{Br}$ sources are together exhibited in Figure 1. As shown in the figure, the main diffraction peaks regarding $\mathrm{BiOBr}-\mathrm{IL}$ and $\mathrm{BiOBr}-\mathrm{SB}$ are similar with almost same peak positions and intensity, but the peaks of $\mathrm{BiOBr}$-IL show dispersion feature to some extent (Figure 1(b)). By contrast, the diffraction peaks of BiOBr-SB look shaper and more refined (Figure 1(a)), though the positions of the main peaks are consistent with those of the other sample. In the $2 \theta$ position of $10.9^{\circ}, 21.9^{\circ}, 25.3^{\circ}, 31.8^{\circ}, 32.3^{\circ}, 39.4^{\circ}, 46.3^{\circ}, 50.8^{\circ}$, and $57.3^{\circ}$, there appear nine peaks, respectively. By comparing with JCPDS database, it can be identified that these peaks coincide with those of BiOBr crystal (PDF Number 73-2061), corresponding to the crystal faces (001), (002), (011), (012), (110), (112), (020), (014), and (212), respectively, which belongs to tetragonal crystal system with space group $\mathrm{P}_{4} \mathrm{nmm}(129)$. 

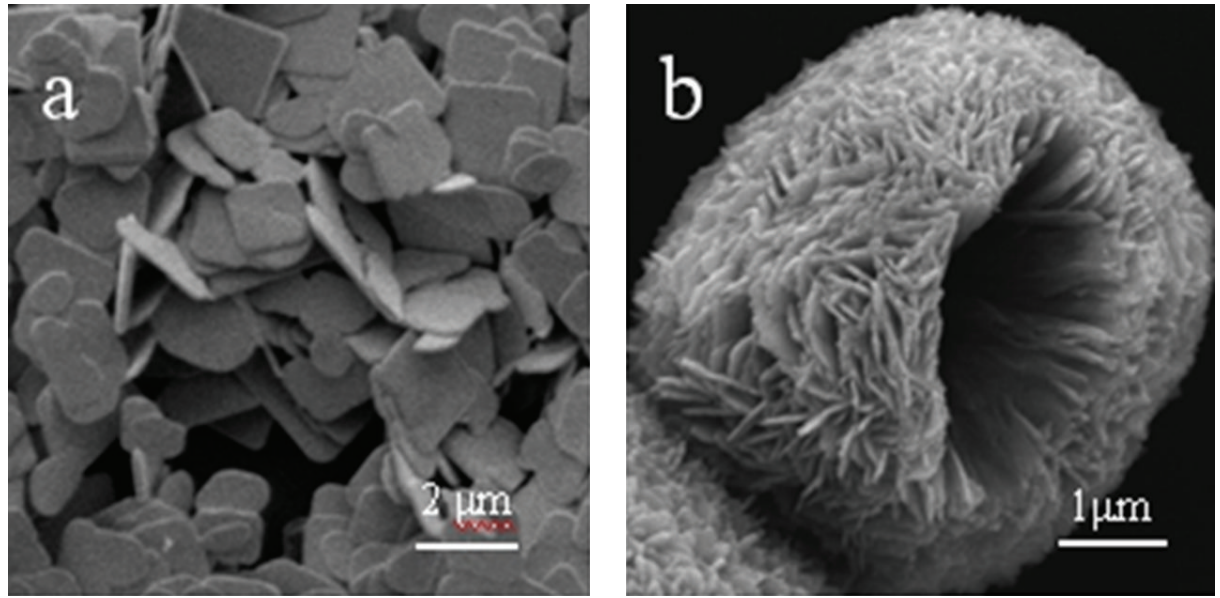

FIGURE 2: The SEM images of as-prepared BiOBr samples. (a) BiOBr-SB; (b) BiOBr-IL.

Moreover, no emergence of extra peaks implies quite elevated purity of as-prepared $\mathrm{BiOBr}$ product samples.

According to Scherrer formula: $d=K \lambda /(B \cos \theta)$, where $\lambda, \theta$, and $B$ are $\mathrm{X}$-ray wavelength, Bragg diffraction angle, and half peak width of (012) face, respectively, the mean particle diameter $(d)$ of both $\mathrm{BiOBr}$ powders can be calculated: $\operatorname{BiOBr}-\mathrm{SB}(d=516 \mathrm{~nm}), \mathrm{BiOBr}-\mathrm{IL}(127 \mathrm{~nm})$, which basically agree with the SEM observation. Obviously, assistance of the ionic liquid could make the $\mathrm{BiOBr}$ crystal grains become smaller, verifying the regulating action of the ionic liquid in this inorganic synthesis [14]. Besides, for BiOBr-IL and $\mathrm{BiOBr}-\mathrm{SB}$, the diffraction peak strength ratios for crystal faces (110) to (012) are 2.71 and 0.98 , respectively, indicating that the ionic liquid contributes to orienting the growth of crystal face (110) in the $\mathrm{BiOBr}$ crystal growth process.

3.2. Morphology and Specific Surface Area of the BiOBr Samples. The SEM images of the BiOBr samples prepared by using different $\mathrm{Br}$ sources are shown in Figure 2. The $\mathrm{BiOBr}-\mathrm{SB}$ crystals mostly show square sheets with about $2 \mu \mathrm{m}$ edge length and $50 \mathrm{~nm}$ thickness. In contrast, the shape of the BiOBr-IL crystals reveals much difference, displaying a spherically porous structure with a hole on it, just like clustered flower petals. This further proves that the ionic liquid has considerable impact on the morphology of asprepared $\mathrm{BiOBr}$ products. The particular microstructure of BiOBr-IL crystals with higher porosity may be partly attributed to higher dielectric constant $(\varepsilon=11.7)$ and good hydrophilicity of the ionic liquid $\left[\mathrm{C}_{4} \mathrm{mim}\right] \mathrm{Br}$, which could effectively retard conglomeration vamong the nascent $\mathrm{BiOBr}$ crystalline grains. Referring to the formation mechanism of CuS hollow spheres [15], it is conceivable for us to speculate the reason while a little hole was formed on the surface of the BiOBr-IL globoids. The speculation is based on the fact that the ionic liquid $\left[\mathrm{C}_{4} \mathrm{mim}\right] \mathrm{Br}$ preferentially clusters together according to a special pattern to form the "crystal nucleus," on which the $\mathrm{BiOBr}$ crystalline plates grow. During washing and cooling, the template could escape from the inside of the core-shell structure, leaving an irregular hole on the outside.

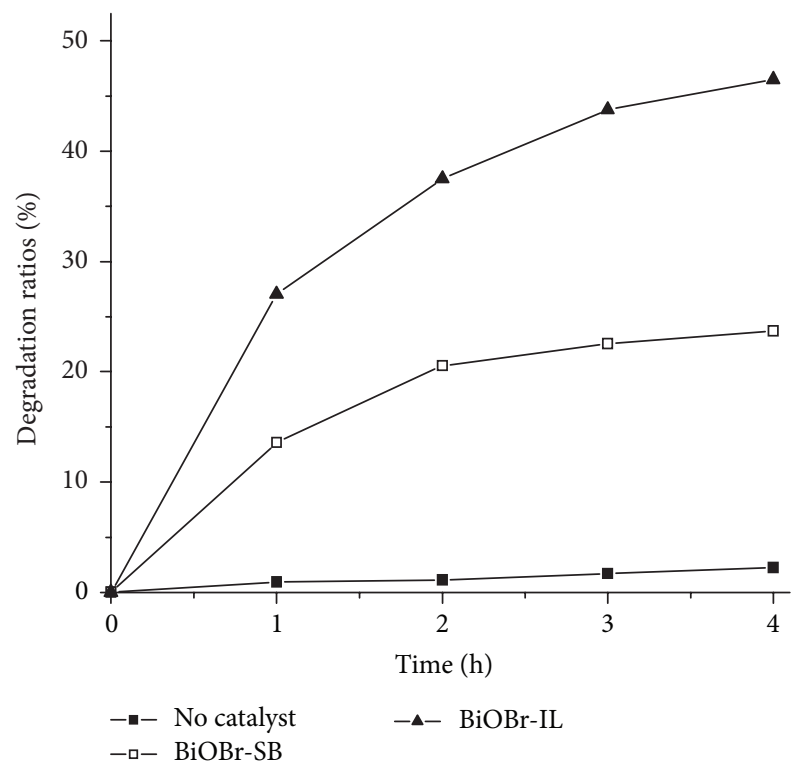

FIGURE 3: The plot of aniline degradation ratio versus reaction time.

The measurements of specific surface area for BiOBr-IL and $\mathrm{BiOBr}-\mathrm{SB}$ are $14.71 \mathrm{~m}^{2} / \mathrm{g}$ and $6.36 \mathrm{~m}^{2} / \mathrm{g}$, respectively.

3.3. Photocatalytic Degradation of Aniline over Both $\mathrm{BiOBr}$ Samples. The removal or degradation of the aniline in wastewater via visible light catalytic action of both $\mathrm{BiOBr}$ microcrystals has been experimentally determined. Just as shown in Figure 3, in the absence of any catalyst, the degradation ratio (ratio between the aniline concentration decrease at a given time and the initial concentration) of the aniline solution is almost negligible despite the sufficiently long visible light irradiation time. This means that suitable catalyst for the degradation reaction is necessary. The BiOBrSB microcrystals have been found to possess visible light catalytic activity to some degree, but the degradation ratio of aniline is not satisfactory. At reaction times of $1 \mathrm{~h}$ and $4 \mathrm{~h}$, 


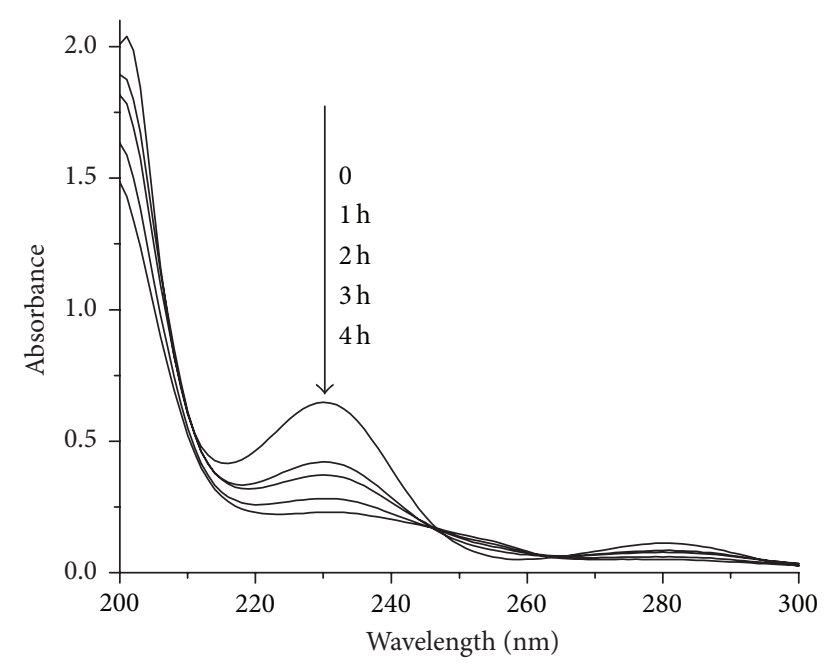

FIgURE 4: The changing relation of UV-vis absorption spectra during the aniline degradation process.

the degradation ratios in the presence of BiOBr-SB microcrystals were $13.60 \%$ and $23.71 \%$, respectively. Compared to $\mathrm{BiOBr}-\mathrm{SB}$, the visible light catalytic activity of $\mathrm{BiOBr}$ IL microcrystals was significantly higher. During the same reaction time as $\mathrm{BiOBr}-\mathrm{SB}$, the corresponding degradation ratios in the presence of BiOBr-IL reached $27.04 \%$ and $46.51 \%$, respectively, of which the top degradation ratio is nearly twice that of $\mathrm{BiOBr}-\mathrm{SB}$. In consideration of the measurement results of SEM and specific surface area, we can deduce the reason for the difference of catalytic ability of the two $\mathrm{BiOBr}$ crystals. The larger specific surface area of the clustered flower petal BiOBr-IL could provide more active sites in comparison with lamellar BiOBr-SB, which should be the main reason for the difference in catalytic activity. What is more, the porous and dispersive microstructure of the BiOBr-IL crystals could make visible light scattered or reflected, leading to extension of the light propagation path, thus generating more photoelectrons and vacancies [16].

In the process of photocatalytic reaction, the color of the aniline solution changed from colorless to brownish yellow, visually depicting the degradation course of aniline. The serial changes of absorption spectra of the aniline solution catalyzed by $\mathrm{BiOBr}-\mathrm{IL}$ crystals during different reaction times are shown in Figure 4. Evidently, the intensity of the characteristic absorption peaks $(\lambda=230 \mathrm{~nm}, \lambda=280 \mathrm{~nm})$ gradually weakens with the prolonging of reaction time, suggesting a gradual reduction of aniline concentration. By measuring the intermediate products during different reaction stages we have verified the existence of such species by GC-MS measurements: aminophenol, nitrobenzene, and azobenzene, and the concentration relationship of these species follows: aminophenol $>$ nitrobenzene $>$ azobenzene. In addition, we also detected inorganic species like $\mathrm{NO}_{3}{ }^{-}$and $\mathrm{CO}_{3}{ }^{2-}$ through ion chromatography (ICS-900, Dionex, USA). Referring to related report [11], we suggest a possible 4-step mechanism for aniline degradation: (1) reactive species, such as radicals, stemming from reaction of photogenerated vacancies with
$\mathrm{H}_{2} \mathrm{O}$ oxidize aniline to generate azobenzene, nitrobenzene, and aminophenol; (2) the intermediates turn to hydroxyl derivatives via electrophilic addition; (3) the aromatic rings of aromatic species break to form 6-carbon aldehydes and acids; (4) the 6-carbon intermediates further degrade to mineralization species: $\mathrm{NO}_{3}{ }^{-}, \mathrm{CO}_{3}{ }^{2-}$.

\section{Conclusions}

(1) Through hydrothermal and solvothermal approach, the square sheet-like and clustered flower petal-like $\mathrm{BiOBr}$ microcrystals were synthesized. The XRD and SEM measurements show that ionic liquid $\left[\mathrm{C}_{4} \mathrm{mim}\right] \mathrm{Br}$ not only acts as reactant to provide a $\mathrm{Br}$ source, but also more importantly can play the role of soft template to fabricate the morphologies of the resulting products. (2) The as-prepared $\mathrm{BiOBr}$ products with both different kinds of morphologies all have visible light catalytic activity in varying degrees, but, in the case of aqueous aniline degradation, the photocatalytic activity of the clustered flower petal-like BiOBr-IL microcrystals was much larger than that of the quadrate lamellar BiOBr-SB products.

\section{Conflict of Interests}

The authors declare that there is no conflict of interests regarding the publication of this paper.

\section{Acknowledgments}

The authors gratefully acknowledge the financial support from the National Natural Science Foundation of China (21576073) and Key Laboratory Construction Project for Chemical Engineering of Mineral Resources of Luoyang City (1003016A).

\section{References}

[1] E. S. Aazam and R. M. Mohamed, "Environmental remediation of direct blue dye solutions by photocatalytic oxidation with cuprous oxide," Journal of Alloys and Compounds, vol. 577, pp. 550-555, 2013.

[2] M. A. Nasalevich, E. A. Kozlova, T. P. Lyubina, and A. V. Vorontsov, "Photocatalytic oxidation of ethanol and isopropanol vapors on cadmium sulfide," Journal of Catalysis, vol. 287, pp. 138-148, 2012.

[3] V. Píšt'ková, M. Tasbihi, M. Vávrová, and U. L. Štangar, "Photocatalytic degradation of $\beta$-blockers by using immobilized titania/silica on glass slides," Journal of Photochemistry and Photobiology A, vol. 305, pp. 19-24, 2015.

[4] D. Zhang, M. Wen, B. Jiang, G. Li, and J. C. Yu, "Ionothermal synthesis of hierarchical $\mathrm{BiOBr}$ microspheres for water treatment," Journal of Hazardous Materials, vol. 211-212, pp. 104-111, 2012.

[5] E. Brillas, E. Mur, R. Sauleda et al., "Aniline mineralization by AOP's: anodic oxidation, photocatalysis, electro-Fenton and photoelectro-Fenton processes," Applied Catalysis B: Environmental, vol. 16, no. 1, pp. 31-42, 1998.

[6] Z. Liu, B. Wu, D. Xiang, and Y. Zhu, "Effect of solvents on morphology and photocatalytic activity of $\mathrm{BiOBr}$ synthesized 
by solvothermal method," Materials Research Bulletin, vol. 47, no. 11, pp. 3753-3757, 2012.

[7] J. Henle, P. Simon, A. Frenzel, S. Scholz, and S. Kaskel, "Nanosized $\mathrm{BiOX}(\mathrm{X}=\mathrm{Cl}, \mathrm{Br}, \mathrm{I})$ particles synthesized in reverse microemulsions," Chemistry of Materials, vol. 19, no. 3, pp. 366373, 2007.

[8] J.-S. Xu, Y.-J. Zhu, and F. Chen, "Microwave-assisted solvothermal ionic liquid rapid synthesis of aluminum fluorohydroxide single-crystalline octahedra," Materials Letters, vol. 94, pp. 104107, 2013.

[9] L. Wu, L. Liao, G. Lv, F. Qin, and Z. Li, "Microstructure and process of intercalation of imidazolium ionic liquids into montmorillonite," Chemical Engineering Journal, vol. 236, pp. 306-313, 2014.

[10] Y. Huang, Y. Xu, Q. He, Y. Cao, and B. Du, "Rapid removal of aniline from contaminated water by a novel polymeric adsorbent," Water Environment Research, vol. 86, no. 1, pp. 20-27, 2014.

[11] C. D. Wu, J. Y. Zhang, L. Wang, and M. H. He, "Removal of aniline and phenol from water using raw and aluminum hydroxide-modified diatomite," Water Science and Technology, vol. 67, no. 7, pp. 1620-1626, 2013.

[12] A. Kumar and N. Mathur, "Photocatalytic degradation of aniline at the interface of $\mathrm{TiO}_{2}$ suspensions containing carbonate ions," Journal of Colloid and Interface Science, vol. 300, no. 1, pp. 244252, 2006.

[13] B. Pare, V. S. Solanki, and P. Singh, "Photocatalytic degradation of Acridine Yellow G and Aniline Blue B dyes in a slurry batch reactor using visible light and $\mathrm{ZnO}$ suspension," Journal of the Indian Chemical Society, vol. 88, no. 10, pp. 1533-1540, 2011.

[14] G. Wang, Z. Ling, C. Li, Q. Dong, B. Qian, and J. Qiu, "Ionic liquid as template to synthesize carbon xerogels by coupling with $\mathrm{KOH}$ activation for supercapacitors," Electrochemistry Communications, vol. 31, pp. 31-34, 2013.

[15] L. Ge, X.-Y. Jing, J. Wang et al., "Ionic liquid-assisted synthesis of $\mathrm{CuS}$ nestlike hollow spheres assembled by microflakes using an oil-water interface route," Crystal Growth \& Design, vol. 10, no. 4, pp. 1688-1692, 2010.

[16] J. G. Yu, Y. R. Su, and B. Cheng, "Template-free fabrication and enhanced photocatalytic activity of hierarchical macro-/mesoporous titania," Advanced Functional Materials, vol. 17, no. 12, pp. 1984-1990, 2007. 

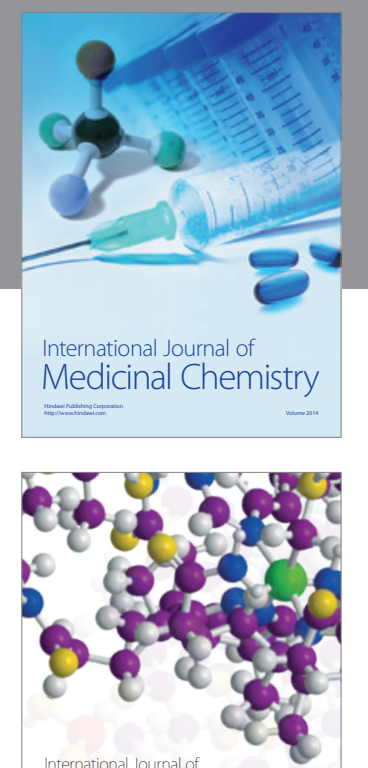

\section{Carbohydrate} Chemistry

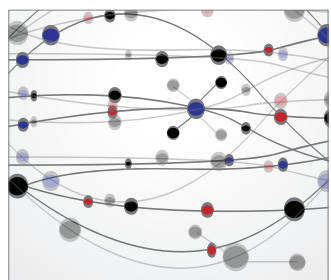

The Scientific World Journal
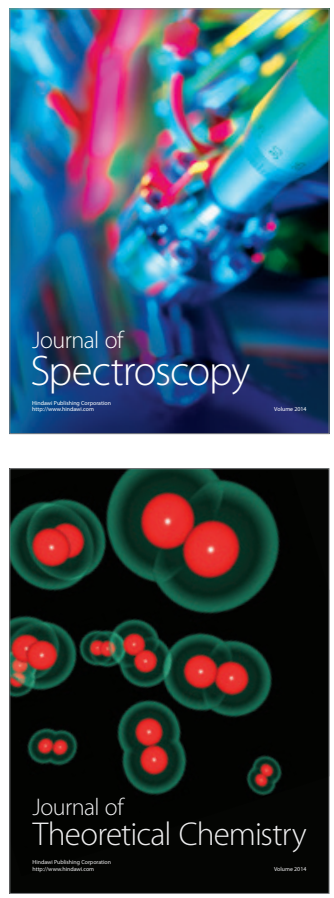
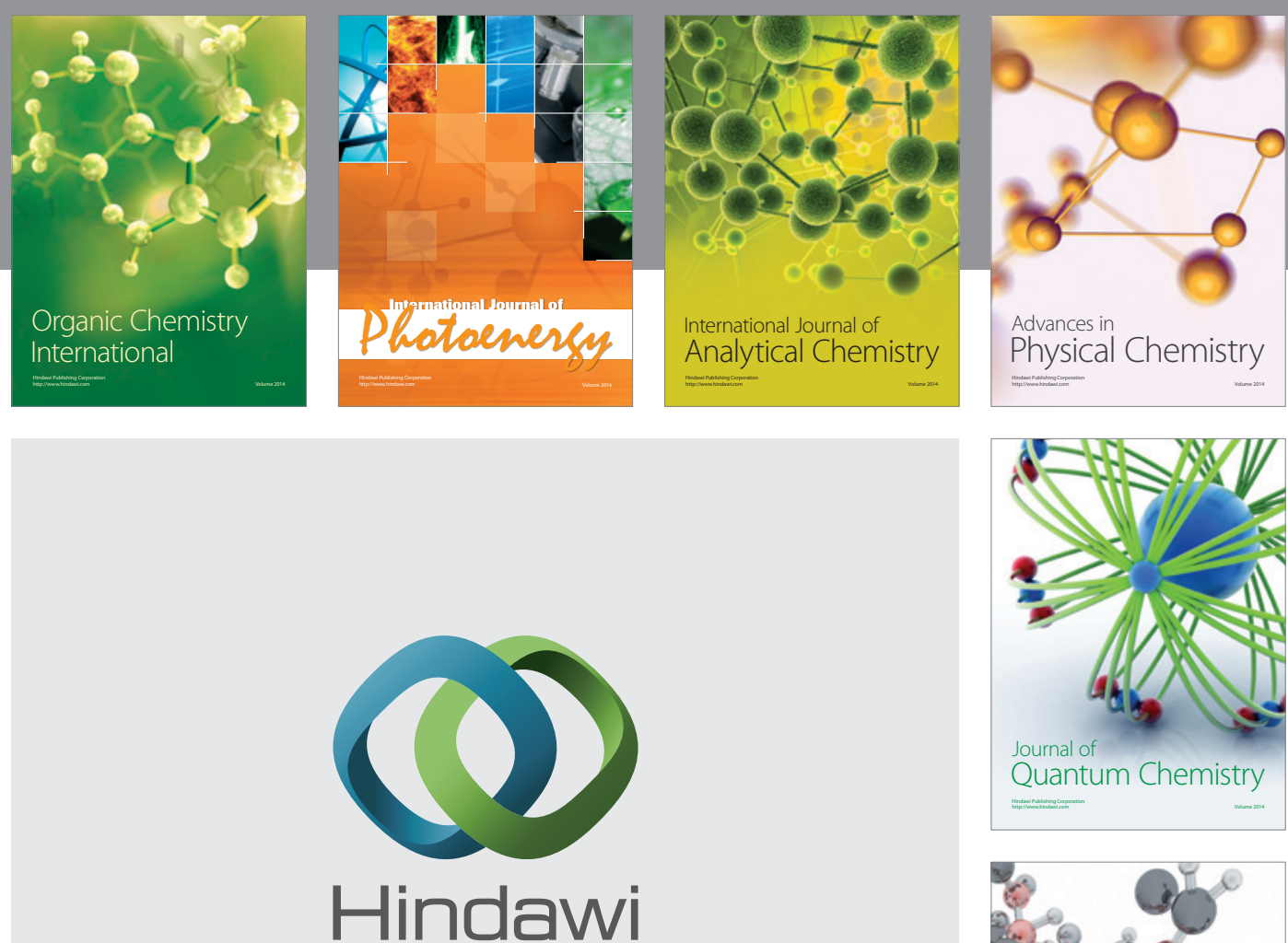

Submit your manuscripts at

http://www.hindawi.com

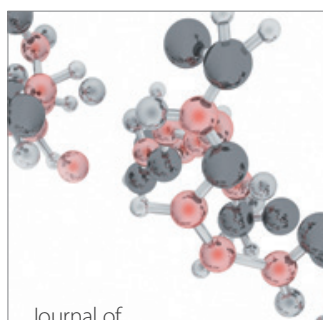

Analytical Methods

in Chemistry

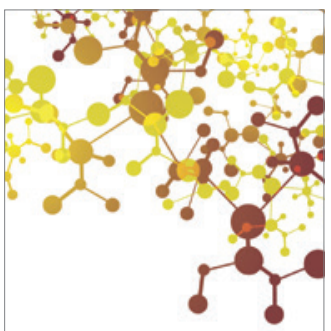

Journal of

Applied Chemistry

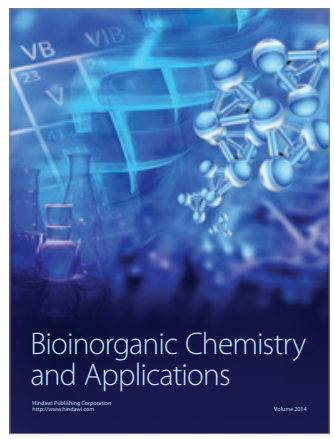

Inorganic Chemistry
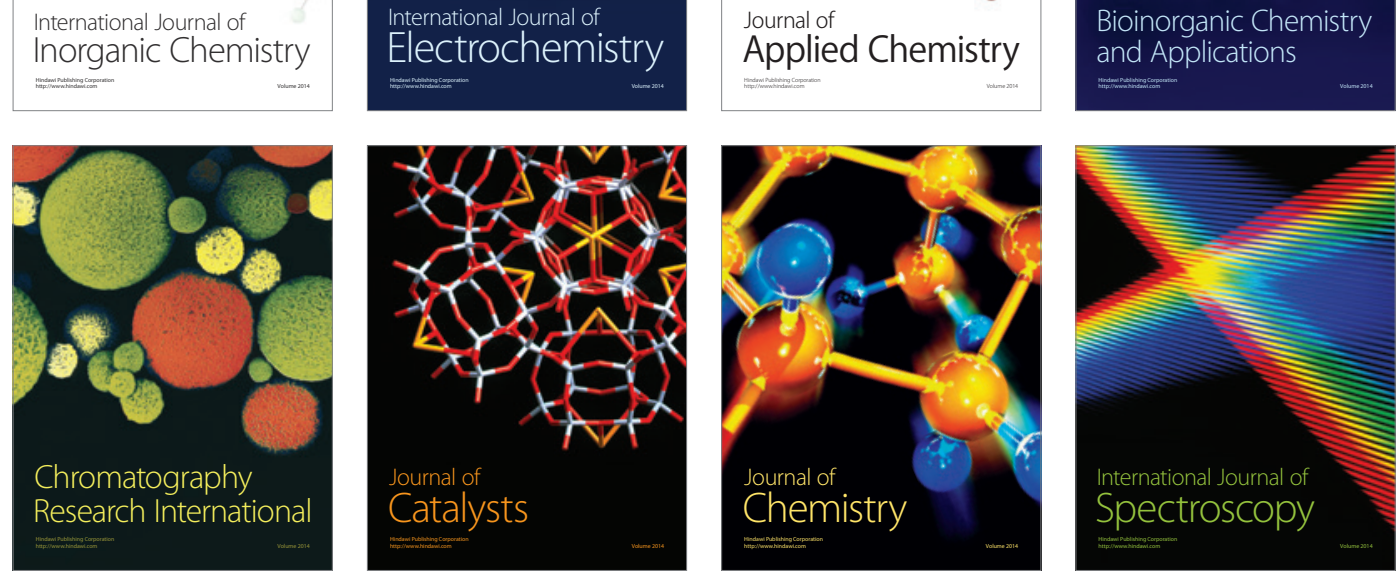